\title{
An acute eccentric exercise increases circulating myomesin 3 fragments
}

\author{
Minjung Lee, Jaehoon Shin, Tatsuya Kato, Kazue Kanda, Satoshi Oikawa, Jun Sakuma, Kaoru Sugama, \\ Yasuo Kawakami, Katsuhiko Suzuki and Takayuki Akimoto*
}

\begin{abstract}
Discovery of blood biomarkers to evaluate exercise-induced muscle damage have attracted many researchers and coaches. This study aimed to determine changes in circulating myomesin 3 fragments as a novel biomarker for exercise-induced muscle damage. Nine healthy males performed 10 sets of 40 repetitions of one-leg calf-raise exercise by the load corresponding to the half of their body weight. Muscle symptoms were evaluated by a visual analog scale (VAS). Blood samples were collected before and 2, 4, 24, 48, 72, and $96 \mathrm{~h}$ post-exercise. Plasma myomesin 3 fragments levels were significantly increased at $96 \mathrm{~h}$ after the eccentric exercise. The myomesin 3 fragments levels were correlated with other biomarkers of muscle damage and the muscle symptoms. These results suggest that the circulating myomesin 3 fragments levels are potential biomarkers reflecting eccentric exercise-induced muscle damage.
\end{abstract}

Keywords: Myomesin, Eccentric exercise, Muscle damage, Biomarkers

\section{Introduction}

Muscle damage is induced not only in pathological setting (i.e., muscular dystrophies) but also physiological setting (i.e., unaccustomed exercise and/or eccentric exercise), causing skeletal myofiber disruption, inflammatory cell infiltration, and muscle soreness [1-5]. There is a growing need for biomarkers in order to monitor disease progression and/or efficiency of treatments in patients with muscular dystrophies. Similarly, researchers in sport sciences have been looking for biomarkers to monitor muscle damage of athletes to plan/change their training since the exercise-induced muscle damage cause various muscle symptoms which can impair sport performance [6].

A number of biomarkers for muscle damage have been discovered so far. Among them, circulating intramuscular proteins, such as creatine kinase (CK) and myoglobin $(\mathrm{Mb})$, are generally used in many clinical diagnoses $[7,8]$. Delayed-onset muscle soreness (DOMS), changes

*Correspondence: axi@waseda.jp

Faculty of Sport Sciences, Waseda University, 2-579-15 Mikajima,

Tokorozawa, Saitama 359-1192, Japan in muscle tenderness, and the range of motion have also been used to indicate muscle symptoms of exerciseinduced muscle damage $[9,10]$. However, researchers continue to seek novel biomarkers for muscle damage to find more sensitive biomarkers that can precisely reflect magnitude of muscle damage and symptoms than the conventional ones [11].

Recent progress in proteomic technology offers a strategy for the comprehensive analysis of protein expression, which can be applied to the search for biomarkers. In fact, several proteomic studies, including ours, have already been reported to identify novel biomarkers for muscle damage in patients with muscle degenerative diseases or in athletes who engaged in eccentric exercise [12-15]. Rouillon et al. [14] revealed that two fragments of a myofibrillar structural protein, myomesin 3 , were significantly increased in sera of patients with muscular dystrophies, who show progressive muscle disruption and regeneration, by using a comprehensive high-resolution mass spectrometry approach. Regarding exercise-induced muscle damage, they reported that the myomesin 3 fragments did not change after an acute bout of eccentric downhill running in mice [14]. However, it 
has not been determined whether the myomesin 3 fragments are responsible to exercise-induced muscle damage among human subjects.

In this study, we measured the levels of the circulating myomesin 3 fragments in healthy volunteers before and after 10 sets of 40 repetitions of eccentric one-leg calfraise exercise. In our knowledge, this is the first study describing a significant increase of the myomesin 3 fragments in response to eccentric exercise.

\section{Methods \\ Subjects}

Nine untrained healthy males participated in the original investigation [16]. The mean $( \pm \mathrm{SD})$ characteristics of the subjects were as follows: age $24.8 \pm 1.3$ years, body mass $62.3 \pm 6.3 \mathrm{~kg}$, and height $1.72 \pm 0.05 \mathrm{~m}$. The subjects had not been involved in any intense exercise or resistance training for at least two weeks before the exercise bout, and were not taking any drugs, nutritional supplements, or participating in recovery strategies, such as massage, stretching, or cryotherapy. The subjects were instructed to maintain their usual daily schedules during the experiment. The study protocol was approved by the Ethics Committee of Waseda University, Japan, in accordance with the Declaration of Helsinki [17], and the subjects gave their written informed consent.

\section{Experimental design}

The subjects performed a calf-raise exercise, including repetitive eccentric muscle contractions, with their right leg on a force plate, as described previously [16]. Briefly, each subject rested on an exercise device specially designed for ankle plantar flexion, with the knee joint extended and the metatarsal bone resting on a stool. The slope of the backrest was $30^{\circ}$, so that the exercise load corresponded to approximately half of the subject's weight (exercise load $=$ body mass $\times$ sin $30^{\circ}$ ). With their right leg, subjects performed single-leg ankle plantar flexion exercise consisting of 10 sets of 40 repetitions with a 3 -min rest between sets. The range of motion (ROM) of the ankle joint during the exercise was maintained between $20^{\circ}$ (dorsiflexion position) and $15^{\circ}$ (plantar flexion position) using an electronic goniometer (SG110/A, Biometrics, Newport, UK) with its ends attached to the distal-lateral part of the fibula and the lateral part of the foot. Each subject received visual feedback on his ankle joint ROM during the exercise via display of the joint ROM value on a personal computer. The exercise was performed in accordance with the rhythm of an electrical metronome at a speed of 60 counts/min; ankle dorsiflexion and plantar flexion were alternated and repeated every $1 \mathrm{~s}$. All subjects completed a total of 400 repetitions of ankle plantar flexion. Delayed-onset muscle soreness (DOMS) was rated with a visual analog scale (VAS): a 100-mm line with "no pain" at one end and "extremely sore" at the other. The tenderness of the exercised muscle correlative to DOMS was assessed using the FP meter (SN402, Navis, Japan) at $1 \mathrm{~kg}$. The point of measurement was the middle point of medial gastrocnemius [16]. Blood samples were collected before and 2, 4, 24, 48, 72 , and $96 \mathrm{~h}$ after exercise. Serum myoglobin $(\mathrm{Mb})$, creatine kinase, $(\mathrm{CK})$ and lactate dehydrogenase (LDH) were measured as described previously $[16,18]$. Data for serum levels of $\mathrm{Mb}, \mathrm{CK}$, and $\mathrm{LDH}$ as well as muscle symptoms (ROM, VAS, and tenderness) were obtained from the previous studies $[16,18]$.

\section{Immunoblotting analysis}

The collected plasma was mixed with complete protein-loading buffer containing $50 \mathrm{mM}$ Tris- $\mathrm{HCl}(\mathrm{pH}$ 6.8), $1 \%$ SDS, $10 \%$ glycerol, $20 \mathrm{mM}$ dithiothreitol, $127 \mathrm{mM}$ 2-mercaptoethanol, and $0.01 \%$ bromophenol blue, supplemented with protease inhibitors (Roche) and phosphatase inhibitors (Sigma-Aldrich, St. Louis, MO, USA). The plasma samples were transferred to microfuge tubes, heated for $1 \mathrm{~min}$ at $95{ }^{\circ} \mathrm{C}$, and centrifuged in a microfuge for $5 \mathrm{~min}$ at $12,000 \times \mathrm{g}$ at room temperature. The plasma samples were then loaded onto $7.5 \%$ gels (depending on the molecular weight of the protein) for SDS-polyacrylamide gel electrophoresis (PAGE), transferred to a nitrocellulose membrane, and immunodetected with an enhanced chemiluminescence kit (ECL prime, Amersham) using the LAS3000 Imaging System (Fuji Film, Tokyo, Japan), as described previously [19]. Antibodies directed against the myomesin 3 fragments were used for the immunoblotting analysis: anti-myomesin 3 (MYOM3) polyclonal antibody (17692-1-AP; Proteintech, Chicago, IL, USA). The secondary antibodies used were horseradish peroxidase (HRP)-conjugated goat anti-rabbit IgG antibody (Amersham). The proteins were quantified with the ImageJ software (NIH, Bethesda, MD, USA).

\section{Statistical analysis}

The data were analyzed with Friedman test. When significant time effects were evident, pairwise comparisons were analyzed by the Dunn's test with Bonferroni's correction. Associations between data were analyzed with Pearson's correlation coefficient (r). Statistical significance was set at $p<0.05$, and the data are presented as means \pm standard error (SE). All statistical analyses were performed by SPSS statistics ver. 26 (IBM, USA). 


\section{Results}

Changes in circulating myomesin 3 fragments after an acute eccentric exercise

We measured the levels of the myomesin 3 fragments in plasma of nine subjects before and after an acute eccentric exercise. The myomesin 3 fragments were detected at $100 \mathrm{kDa}$ and $130 \mathrm{kDa}$ (Fig. 1a), respectively, as previously described [14]. We found that the myomesin 3 fragments increased significantly $96 \mathrm{~h}$ after the exercise (Fig. 1b, $p<0.05$ ), although the changes in the circulating myomesin 3 levels varied among subjects (Additional file 1: Fig. S1).

\section{Relationships between the myomesin $\mathbf{3}$ fragments levels and other blood markers of muscle damage}

As shown in Table 1 and Fig. 2a, there were positive correlations between the percent changes in the myomesin 3 fragments levels and that in CK $(R=0.602$, $p<0.01$, See also Additional file 2: Table S1), $\mathrm{Mb}$ $(R=0.464, p<0.01$, See also Additional file 2: Table S2), and LDH $(R=0.674, p<0.01$, See also Additional file 2 : Table S3) levels (Table1, Fig. 2a).

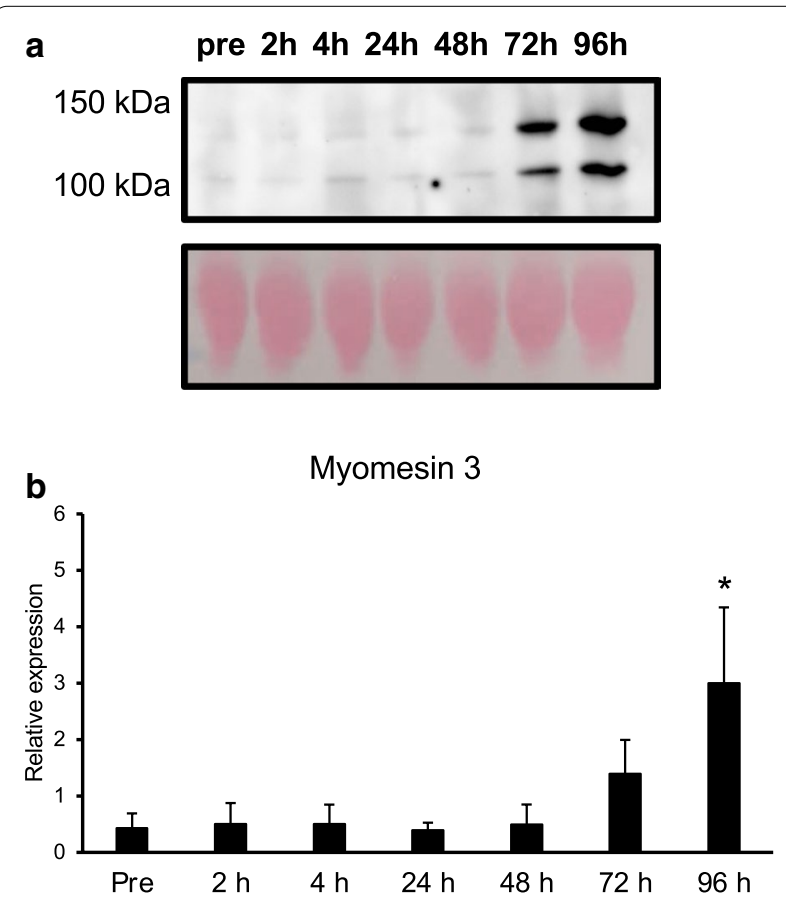

Fig. 1 Changes in circulating myomesin 3 fragments in response to eccentric exercise. a Representative images of western blot for myomesin 3 and Ponceau S staining. b Relative expression of plasma myomesin 3 fragments levels of pre, 2, 4, 24, 48, 72, and $96 \mathrm{~h}$ after an acute bout of eccentric exercise. Values are expressed as means \pm SE $(n=9) .{ }^{*} p<0.05$ vs Pre

\begin{tabular}{lccc}
$\begin{array}{l}\text { Table } 1 \\
\text { of myomesin } \mathbf{3} \text { and }\end{array}$ & $\begin{array}{c}\text { correlation } \\
\text { muscle damage }\end{array}$ & $\begin{array}{c}\text { coefficient } \\
\text { MYOM3 }\end{array}$ & matrix \\
\hline CK & Mb & LDH \\
\hline MYOM3 & $0.602^{* *}$ & $0.464^{* *}$ & $0.674^{* *}$ \\
$\mathrm{CK}$ & & $0.810^{* *}$ & $0.944^{* *}$ \\
$\mathrm{Mb}$ & & & $0.793^{* *}$ \\
$\mathrm{LDH}$ & & & \\
\hline
\end{tabular}

Myomesin 3 (MYOM3), creatine kinase (CK), myoglobin (Mb), lactate dehydrogenase $(\mathrm{LDH})$. All data are calculated as changes for the pre-exercise values. ${ }^{* *} p<0.01$

Relationships between the myomesin 3 fragments levels and muscle symptoms

We next determined whether the changes in the circulating myomesin 3 levels associated with muscle symptoms, such as muscle tenderness (TDN), DOMS, and ROM. As shown in Table 2 and Fig. 2b, we observed positive correlations between percent changes in myomesin 3 levels and that in TDN $(R=0.539, p<0.01$, See also Additional file 2: Table S4) and VAS (visual analog scale for DOMS, $R=0.335, p<0.05$, See also Additional file 2: Table S5). Changes in $\operatorname{ROM}(R=0.038, p=0.838)$ was not correlated with myomesin 3 levels (Table 2, Fig. 2b, See also Additional file 2: Table S6).

\section{Discussion}

The major finding of this study is that circulating myomesin 3 fragments increased in response to an acute bout of eccentric exercise. To our knowledge, this is the first report describing an increase of the myomesin 3 fragments after eccentric exercise in humans.

There are three isoforms of myomesin: myomesin 1, myomesin 2 (M-protein), and myomesin 3. These myomesins are the principal components of the cytoskeletal structure called the M-band that cross-links antiparallelly arranged myosin filaments and titin filaments in the middle of the sarcomere $[20,21]$. It has been suggested that myomesin is a molecular spring whose elasticity guards the stability of the sarcomere similar to titin [22]. Expression of myomesin 3 was found mainly in type IIa fibers of skeletal muscle, while type IId/x fibers express more myomesin 2 and myomesin 1 is expressed in all muscle fibers [22]. Since type II muscle fibers are more susceptible to eccentric exercise-induced muscle damage [23], it seems to be reasonable that type II fibers were selectively disrupted and the myomesin 3 fragments were released into blood stream in response to eccentric exercise.

Rouillon et al. [14] revealed that two fragments of myomesin 3 are detected in sera of patients with 
a
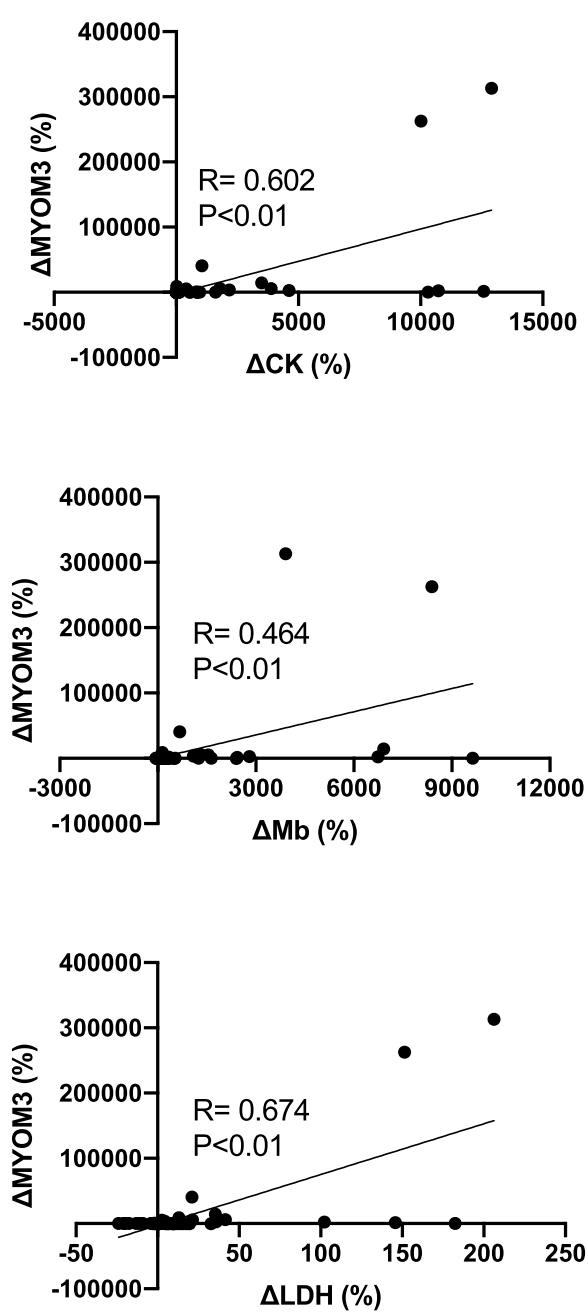

b Muscle symptoms
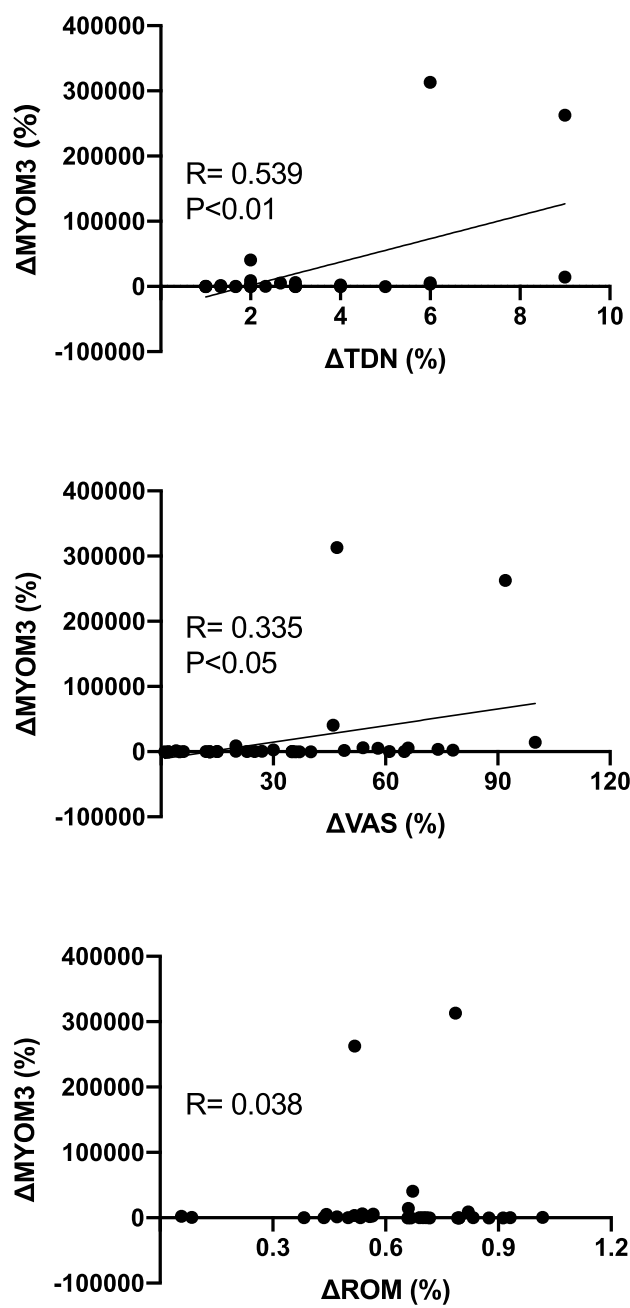

Fig. 2 Relationships between myomesin 3 fragments levels and circulating biomarkers of muscle damage, and muscle symptoms after eccentric exercise. a Relationships between Myomesin 3 (MYOM3) vs Creatine kinase (CK), lactate dehydrogenase (LDH), and myoglobin (Mb). b Relationships between Myomesin 3 (MYOM3) vs ankle range of motion (ROM) in dorsal flexion, Muscle tenderness (TDN), and visual analog scale (VAS) for delayed-onset muscle soreness (DOMS). All data are percent changes $(\Delta)$ between pre-exercise and post-exercise values. ${ }^{*} p<0.05$, ${ }^{* *} p<0.01$

Table 2 Pearson's correlation coefficient matrix of myomesin 3 and muscle symptoms

\begin{tabular}{|c|c|c|c|c|}
\hline & MYOM3 & TDN & VAS & ROM \\
\hline MYOM3 & & $0.539^{* *}$ & $0.335^{*}$ & 0.038 \\
\hline TDN & & & $0.813^{* *}$ & -0.127 \\
\hline VAS & & & & $-0.451^{* *}$ \\
\hline ROM & & & & \\
\hline
\end{tabular}

Myomesin 3 (MYOM3), muscle tenderness (TDN), and visual analog scale (VAS) for delayed-onset muscle soreness (DOMS), and ankle range of motion (ROM) in dorsal flexion. All data are calculated as changes for the pre-exercise values. ${ }^{*} p<0.05,{ }^{* *} p<0.01$ muscular dystrophies as well as model mice for muscular dystrophy ( $\mathrm{mdx}$ mouse), while myomesin 3 fragments are barely detected in sera of healthy individuals and wild-type animals in their original investigation. Then, Rouillon et al. [14] further tested if an acute bout of eccentric exercise alters circulating levels of the myomesin 3 fragments in mice. They found that the levels of the myomesin 3 fragments did not change after an acute eccentric downhill running exercise in mice. In contrast, we found that an acute eccentric calf-raise exercise increased the myomesin 3 fragments in humans. To explain the different behavior of the myomesin 3 fragments in mice and humans, we suggest 
that the degree of muscle damage was different among these studies. It is possible that the eccentric downhill running exercise was not sufficient to induce muscle damage in mice because the serum CK levels showed no change in mice [14], while we found a significant increase in $\mathrm{CK}$ after the eccentric calf-raise exercise in the present study.

Rouillon et al. also reported that circulating levels of myomesin 3 showed lower inter-individual variances than those of CK among patients with muscular dystrophies [14]. On the other hand, we observed that inter-individual variances still existed in the myomesin 3 levels similar to the other biomarkers for muscle damage in this study. Muscular dystrophy patients have identical genetic factors that might cause less diversity of muscle damage, while healthy subjects may have less in common than muscular dystrophy patients and have more various factors influencing exercise-induced muscle damage. Firstly, genetic polymorphisms in sarcomeric proteins and growth factors might vary the degree of exercise-induced muscle damage among the healthy subjects $[24,25]$. Also, considering that fast-twitch fibers show severer eccentric contraction-induced muscle damage than slow-twitch fibers [23], distinct composition of fast-twitch muscle fiber in each participant might have influence on the degree of exercise-induced muscle damage.

There was no clear difference in the time course of cytosolic $\mathrm{CK}, \mathrm{LDH}, \mathrm{Mb}$, and structural myomesin 3 appearance in the circulation after the eccentric exercise, despite the different roles and distributions in muscle cells. The reason for the similar kinetics of these biomarkers in the circulation is that the mechanisms via which each muscle damage marker leaks into the blood may be the same [26]. Generally, damage to the sarcolemma causes intracellular proteins to leak into the blood, which is a major cause of the increase in cytosolic proteins in the blood following eccentric exercise [26].

The present study has limitations. Various factors, such as gender, age, muscle mass, genetic factors, selected muscle groups, training frequency, and frequency of daily exposure to eccentric contractions, cause differences in exercise-induced muscle symptoms among individuals $[26,27]$. We could not avoid the subjects being exposed to eccentric contractions on a daily basis although the subjects in this study performed limited resistance training during the experimental period.

In conclusion, an increase in the plasma myomesin 3 fragments is potentially a biomarker of exercise-induced muscle damage because it reflects the changes that occur in other markers of muscle damage and in the muscle symptoms observed after eccentric exercise.

\section{Supplementary Information}

The online version contains supplementary material available at https://doi. org/10.1186/s12576-021-00789-y.

Additional file 1: Figure S1. Entire images of Western blot for fragmented myomesin 3. Entire images of Western blot for myomesin 3 and Ponceau S staining. Pre, 2, 4, 24, 48, 72, and $96 \mathrm{~h}$ after an acute bout of eccentric exercise.

Additional file 2: Table S1. Pearson's correlation coefficient matrix of myomesin 3 and creatinine kinase at each time point. Table S2. Pearson's correlation coefficient matrix of myomesin 3 and myoglobin at each time point. Table S3. Pearson's correlation coefficient matrix of myomesin 3 and lactate dehydrogenase at each time point. Table S4. Pearson's correlation coefficient matrix of myomesin 3 and muscle tenderness at each time point. Table S5. Pearson's correlation coefficient matrix of myomesin 3 and DOMS at each time point. Table S6. Pearson's correlation coefficient matrix of myomesin 3 and ROM at each time point.

Acknowledgments

The authors thank the volunteers who participated in this study.

Authors' contributions

Individual author contributions were as follows: conception and design of the experiments (ML, YK, KS (Katsuhiko Suzuki), and TA); data collection (ML, JS,

TK, KK, KS (Kaoru Sugama), JS, and SO); analysis and interpretation of data (ML and TA); drafting the article and revising it critically for important intellectual content (ML and TA); supervision of the study (YK, KS (Katsuhiko Suzuki), and TA). All the authors read and approved the final manuscript.

\section{Funding}

This study was supported in part by Grants-in-Aid for Scientific Research (B) (21300217 to YK) from the Ministry of Education, Culture, Sports, Science and Technology, Japan, and Total conditioning research project (to TA) from Japan Sports Council. ML was supported by the Japan Society for the Promotion of Science.

\section{Availability of data and materials}

The datasets generated and/or analyzed during the current study are included in this study and previous studies $[16,18]$.

\section{Ethics approval and consent to participate}

The research protocol was accepted by Ethics Committee of Waseda University, and completed in accordance with the Declaration of Helsinki [17].

Consent for publication

Not applicable.

\section{Competing interests}

The authors declare that they have no competing interest.

Received: 10 October 2020 Accepted: 4 January 2021

Published online: 19 January 2021

References

1. Bell CD, Conen PE (1968) Histopathological changes in Duchenne muscular dystrophy. J Neurol Sci 7:529-544

2. Fridén J, Lieber RL (1992) Structural and mechanical basis of exerciseinduced muscle injury. Med Sci Sports Exer 24:521-530

3. Howell JN, Chleboun G, Conatser R (1993) Muscle stiffness, strength loss, swelling and soreness following exercise-induced injury in humans. J Physiol 464:183-196

4. Beaton LJ, Tarnopolsky MA, Phillips SM (2002) Variability in estimating eccentric contraction-induced muscle damage and inflammation in humans. Can J Appl Physiol 27(5):516-526 
5. Smith C, Kruger MJ, Smith RM, Myburgh KH (2008) The inflammatory response to skeletal muscle injury: illuminating complexities. Sports Med 38:947-969

6. Highton JM, Twist C, Eston RG (2009) The effects of exercise-induced muscle damage on agility and sprint running performance. J Exercise Sci Fitness 7:24-30

7. Mokuno K, Riku S, Sugimura K, Takahashi A, Kato K, Osugi S (1987) Serum creatine kinase isoenzymes in Duchenne muscular dystrophy determined by sensitive enzyme immunoassay methods. Muscle Nerve 10:459-463

8. Brancaccio P, Maffulli N, Buonauro R, Limongelli FM (2008) Serum enzyme monitoring in sports medicine. Clin Sports Med 27:1-vii

9. Clarkson PM, Nosaka K, Braun B (1992) Muscle function after exerciseinduced muscle damage and rapid adaptation. Med Sci Sports Exerc 24:512-520

10. Eston RG, Finney S, Baker S, Baltzopoulos V (1996) Muscle tenderness and peak torque changes after downhill running following a prior bout of isokinetic eccentric exercise. J Sports Sci 14:291-299

11. Nosaka K, Newton M, Sacco P (2002) Delayed-onset muscle soreness does not reflect the magnitude of eccentric exercise-induced muscle damage. Scand J Med Sci Sports 12:337-346

12. Rouillon J, Zocevic A, Leger T, Garcia C, Camadro JM, Udd B, Wong B, Servais L, Voit T, Svinartchouk F (2014) Proteomics profiling of urine reveals specific titin fragments as biomarkers of Duchenne muscular dystrophy. Neuromuscul Disord 24:563-573

13. Hathout Y, Brody E, Clemens PR, Cripe L, DeLisle RK, Furlong P, GordishDressman $\mathrm{H}$, Hache L, Henricson E, Hoffman EP, Kobayashi YM, Lorts A, Mah JK, McDonald C, Mehler B, Nelson S, Nikrad M, Singer B, Steele F, Sterling D, Sweeney HL, Williams S, Gold L (2015) Large-scale serum protein biomarker discovery in Duchenne muscular dystrophy. Proc Natl Acad Sci USA 112:7153-7158

14. Rouillon J, Poupiot J, Zocevic A, Amor F, Léger T, Garcia C, Camadro JM, Wong B, Pinilla R, Cosette J, Coenen-Stass AM, Mcclorey G, Roberts TC, Wood MJ, Servais L, Udd B, Voit T, Richard I, Svinartchouk F (2015) Serum proteomic profiling reveals fragments of MYOM3 as potential biomarkers for monitoring the outcome of therapeutic interventions in muscular dystrophies. Hum Mol Genet 24:4916-4932

15. Kanda K, Sakuma J, Akimoto T, Kawakami Y, Suzuki K (2017) Detection of titin fragments in urine in response to exercise-induced muscle damage. PLoS ONE 12:e0181623
16. Kanda K, Sugama K, Hayashida H, Sakuma J, Kawakami Y, Miura S, Yoshioka H, Mori Y, Suzuki K (2013) Eccentric exercise-induced delayed-onset muscle soreness and changes in markers of muscle damage and inflammation. Exerc Immunol Rev 19:72-85

17. The Helsinki Declaration of the World Medical Association (WMA) (2014) Ethical principles of medical research involving human subjects. Pol Merkur Lekarski 36:298-301

18. Kanda K, Sugama K, Sakuma J, Kawakami Y, Suzuki K (2014) Evaluation of serum leaking enzymes and investigation into new biomarkers for exercise-induced muscle damage. Exerc Immunol Rev 20:39-54

19. Wada S, Kato Y, Okutsu M, Miyaki S, Suzuki K, Yan Z, Schiaffino S, Asahara H, Ushida T, Akimoto T (2011) Translational suppression of atrophic regulators by microRNA-23a integrates resistance to skeletal muscle atrophy. J Biol Chem 286:38456-38465

20. Agarkova I, Perriard JC (2005) The M-band: an elastic web that crosslinks thick filaments in the center of the sarcomere. Trends Cell Biol 15:477-485

21. Lange S, Himmel M, Auerbach D, Agarkova I, Hayess K, Fürst DO, Perriard JC, Ehler E (2005) Dimerisation of myomesin: implications for the structure of the sarcomeric M-band. J Mol Biol 345:289-298

22. Schoenauer R, Lange S, Hirschy A, Ehler E, Perriard JC, Agarkova I (2008) Myomesin 3, a novel structural component of the M-band in striated muscle. J Mol Biol 376:338-351

23. Fridén J, Sjöström M, Ekblom B (1983) Myofibrillar damage following intense eccentric exercise in man. Int J Sports Med 4:170-176

24. Clarkson PM, Hoffman EP, Zambraski E, Gordish-Dressman H, Kearns A, Hubal M, Harmon B (1985) Devaney JM (2005) ACTN3 and MLCK genotype associations with exertional muscle damage. J Appl Physiol 99:564-569

25. Devaney JM, Hoffman EP, Gordish-Dressman H, Kearns A, Zambraski E (1985) Clarkson PM (2007) IGF-II gene region polymorphisms related to exertional muscle damage. J Appl Physiol 102:1815-1823

26. Brancaccio P, Maffulli N, Limongelli FM (2007) Creatine kinase monitoring in sport medicine. Br Med Bull 81-82:209-230

27. Hyldahl RD, Chen TC, Nosaka K (2017) Mechanisms and Mediators of the Skeletal Muscle Repeated Bout Effect. Exerc Sport Sci Rev 45:24-33

\section{Publisher's Note}

Springer Nature remains neutral with regard to jurisdictional claims in published maps and institutional affiliations.
Ready to submit your research? Choose BMC and benefit from:

- fast, convenient online submission

- thorough peer review by experienced researchers in your field

- rapid publication on acceptance

- support for research data, including large and complex data types

- gold Open Access which fosters wider collaboration and increased citations

- maximum visibility for your research: over $100 \mathrm{M}$ website views per year

At BMC, research is always in progress.

Learn more biomedcentral.com/submissions 